(n)

Art, Illustration and Visual Culture

in Infant and Primary Education

\title{
Aprendizaje de expresión plástica y cultura visual en los nuevos grados universitarios de maestro en educación infantil y educación primaria
}

Rafael Sumozas rafael.sumozas@uclm.es Universidad de Castilla-La Mancha, España

Reference

Sumonaz, Rafael; (2012) "Aprendizaje de expresión plástica y cultura visual en los nuevos grados universitarios de maestro en educación infantil y educación primaria", p. 83-87 . In: Barbosa, Helena; Quental, Joana [Eds]. Proceedings of the 2nd International Conference of Art, Illustration and Visual Culture in Infant and Primary Education. São Paulo: Blucher, 2015. ISSN 2318-695X ISBN: 978-989-98185-0-7 DOI 10.5151/edupro-aivcipe-17

Resumen

Palabras clave

Abstract

Keywords
El objetivo de esta comunicación es evaluar la situación de las enseñanzas del área de expresión plástica en los nuevos grados universitarios de maestro en educación infantil y primaria. El origen de la disciplina expresión plástica y visual se encuentra en los primeros niveles educativos, infantil y primaria, por lo que los estudiantes de magisterio tienen que aprender cómo hay que enseñar educación artística en la escuela y el docente de los nuevos grados, desarrollar una formación adecuada en el futuro maestro de plástica. Para ello, primeramente se analiza el contexto de la enseñanza de la expresión plástica en la universidad, en un segundo lugar se evalúa la enseñanza de la educación artística en el área didáctica de la expresión plástica, para finalmente poder evaluar la metodología y los recursos utilizados en el área.

Aprendizaje, Educación Artística, Nuevos Grados Universitarios, Infantil, Primaria

The aim of this paper is evaluate the status of teaching in the area of artistic expression in the new college degrees in early childhood education teacher and elementary school. The origin of the discipline and visual artistic expression is in the early levels of education, kindergarten and primary, so that student teachers have to learn how to teach art education in school and the teacher of the new degrees, develop a appropriate training future teachers in arts education. For this, first we analyze the context of teaching art education in college. Secondly we evaluate of the teaching of arts education in the educational area of artistic expression, to finally evaluate the methodology and resources used in the area.

Learning, Art Education, New University Degrees, Childhood Education, Primary Education

\section{Introducción}

El aprendizaje de expresión plástica y cultura visual, se inserta oficialmente en España en el Área de conocimientos se denominada Didáctica de la Expresión Plástica y su origen como disciplina se encuentra en educación infantil y primaria, pues los docentes que deben impartir educación plástica deben formase previamente en artes visuales, "las niñas y los niños tienen que cursar en la escuela la asignatura dibujo, sus profesoras y profesores tienen que aprender cómo hay que enseñar a dibujar en la escuela", esta afirmación junto a esta otra: "si no existiera en la escuela primaria... la asignatura de plástica no existiría en la Universidad el área de Didáctica de la Expresión Plástica”, es fundamental para determinar el aprendizaje de expresión plástica y cultura visual. Marín, R. (2008:236). El objetivo básico del área de Didáctica de la Expresión Plástica en los grados de maestro en educación Infantil y primaria es desarrollar una formación adecuada del futuro maestro en educación artística.

Afortunadamente la producción científica sobre Educación Artística ha sido muy fructífera desde la década de los noventa en España, cuando que se incorporaron las nuevas tecnologías 
gresso Internacional

de Arte, llustração e Cultura Visual

na Educação Infantil e Primária

a la educación, como la fotografía, los medios informáticos y el espacio virtual con internet, sobre este tema, la tesis doctoral de María Acaso (1998a): Nuevas tecnologías en la didáctica de la expresión plástica: el CD-ROM como alternativa multimedia a los métodos tradicionales de educación artística, constató la importancia de la incorporación de las nuevas tecnologías a la educación artística, como han hecho otros países de nuestro entorno, además, en la actualidad, el docente del área de educación artística cuenta para el desarrollo de la enseñanza, con tendencias pedagógicas y herramientas organizativas, como la Teoría de la Elaboración de Reigeluth, desarrollada por Acaso (1998b) en: "La teoría de la elaboración como estrategia organizativa dentro del marco de la educación artística como disciplina" la cual considera como la más adecuada para la educación artística como disciplina. En la misma línea Carlos Escaño (2010), constata la evolución de Internet y su repercusión en el contexto de la enseñanza artística por la estrecha relación entre Educación, Arte e Internet. Por otra parte, en relación a la necesidad de desarrollar la expresión plástica, Acaso (2000), en su estudio: "Simbolización, expresión y creatividad" analizó los principales autores en estas líneas de investigación: Piaget, Read y Lowenfeld y propuso tres tipos de procesos que justifican la necesidad de desarrollar la expresión plástica en el niño: un proceso de simbolización, un proceso de desarrollo expresivo y un proceso de desarrollo creativo, este proceso de transmisión del conocimiento, según Acaso (2005a): se realiza con "El currículum oculto visual", mediante el cual el niño "aprende a obedecer a través de la imagen". En los últimos tiempos está proliferando la presencia de las artes en la escuela, como constata Imanol Aguirre (2009): "Sobre los usos del arte" y su utilización en la educación contemporánea y especialmente en esta etapa de la educación infantil, sobre el tema Juan Carlos Arañó en su artículo "Arte, educación y creatividad" (1994), plantea el concepto de creatividad en las artes visuales, e intenta dar una valoración objetiva de la creatividad en las artes plásticas teniendo como referencia previa, considerar los conceptos de realidad, belleza, naturaleza, conocimiento, producción, novedad, libertad y futuro entre otros y la relación de estos conceptos con el de creatividad. Según Carlos Escaño (1999), en la era postmoderna se ofrecen con la televisión, el cine y la informática, creaciones audiovisuales sugestivas que constituyen una nueva realidad que se convierte en replicante, establecido una fuerte competencia con el cosmos humano, que se relaciona de forma directa con la educación, en emociones y en sentimientos, lo que Escaño llama "una vida paralela que se convierte por un -paradójico- ser humano en todo un modus vivendi". Es evidente que existen lazos de unión entre el arte, la educación y la creatividad, Rosario Gutiérrez (2002) defiende la educación artística como un área en la que la creatividad tiene la posibilidad y la obligación de existir. En este sentido considera que "cuando la educación visual y plástica no se plantea sólo al servicio de otras áreas, sino como medio para lograr los fines propios del arte como materia del currículum escolar, es decir, para promover, parafraseando a Eisner (1995), "el desarrollo de la visión artística", estamos asistiendo también al desarrollo del pensamiento creativo por ser éste parte integrante de la experiencia de producción que se realiza en torno al arte".

En el proceso de aprendizaje de expresión plástica, tiene una vital importancia la obra de Luisa María Martínez, Rosario Gutiérrez y Carlos Escaño (coords.) (2008): Nuevas propuestas de acción en educación educativa, donde realizan un análisis de la intervención educativa que se desarrolla en torno a la Educación Artística en los distintos conceptos de la enseñanza formal y no formal, y ponen de manifiesto que este campo de conocimiento no se sustenta ni puede construirse sobre intuiciones, sino que requiere de la indagación crítica propia de cualquier disciplina. De igual modo, es importante la obra de Ricardo Marín (coord.) (2003): Didáctica de la educación artística para primara, en la que profesionales sobre la educación artística abordan los problemas de la enseñanza y el aprendizaje de las artes visuales en los contextos escolares. Esta obra con un enfoque panorámico y plural de las principales ideas, temas e imágenes que pueden contribuir a la mejora de la calidad de las enseñanzas artísticas, el mismo autor, publicó en (2005): Investigación en educación artística: temas, métodos y técnicas de indagación sobre el aprendizaje y la enseñanza de las artes y culturas visuales, donde se ofrece una perspectiva general sobre la educación artística, así como los fundamentos epistemológicos y debates contemporáneos sobre el área, además, recoge los distintos enfoques metodológicos, estrategias y técnicas de investigación, así como temas emergentes, como las competencias básicas, nuevos medios y contextos educativos. 
2nd International Conference

Art, Illustration and Visual Culture

in Infant and Primary Education
2 Congreso Internacional

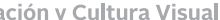
en Educación Infantil y Primaria
Congresso Internacional

de Arte, Ilustração e Cultura Visual

na Educação Infantil e Primária

\section{La Educación Artística en lo nuevos grados universitarios del EEES}

Para la adaptación de la antigua Diplomatura en Magisterio al Espacio Europeo de Educación Superior (EEES), en los últimos años se ha realizado en España una importante labor de adaptación a los presupuestos de Bolonia. Los estudios realizados por (Maldonado, 2004:67) para la ANECA (Agencia Nacional de Evaluación de la Calidad y la Acreditación en España) sobre la inserción laboral de sus titulados en el Libro blanco para el actual Título de grado en magisterio, valoraban las competencias comunes a los cuatro perfiles de inserción laboral de los antiguos egresados de las Diplomaturas de Maestro, según este estudio, se debía partir, en primer lugar, del reconocimiento de algunos graduados, que sólo tenían competencias docentes de carácter general en Educación Infantil (Maestros de Educación Infantil, con responsabilidad en todas las áreas del currículo en este nivel) y en Educación Primaria (Maestros de Educación Primaria, en Matemáticas, Lengua, Conocimiento del Medio y Educación Plástica). En segundo lugar, se debía reconocer que a los graduados (Educación Musical, Educación Física, Lengua Extranjera) competencias profesionales tanto en las materias que daban nombre a su título, como a las materias en las que no estaban especializados (Matemáticas, Lengua, Conocimiento del Medio y Educación Plástica), con lo que vemos que no se formaba específicamente a los estudiantes en Educación Plástica. Por esto, es imprescindible recordar que tanto la LOGSE (1990) (Ley Orgánica de Ordenación General del Sistema Educativo de España) como la LOCE (2002) (Ley Orgánica de Calidad de la Educación de España) establecían que la docencia en Educación Infantil debía ser impartida por profesores con la especialización correspondiente, como sucedía con las áreas de Educación Musical, Educación Física y Lengua Extranjera. También se establecía que los Maestros de Educación Especial y/o Audición y Lenguaje podían acceder a puestos específicos de este ámbito. Además, todos los maestros tenían reconocida, por dichas leyes, la capacidad de docentes en áreas de Educación Primaria: Matemáticas, Lengua, Ciencias- Geografía e Historia y Educación Artística (Plástica), puesto que no existen puestos específicos para la docencia de estas materias. Por esa razón, las autoridades de las distintas Comunidades Autónomas espalolas han venido convocando plazas de maestro para el doble desempeño de tareas de especialista y/o de generalista.

Las enseñanzas artísticas que se están llevando a cabo en la formación de las especialidades de maestros, se han visto considerablemente reducidas con las adaptaciones a Grados (EEES), al punto que algunos especialistas sobre la materia consideran que la presencia de la Educación Artística simplemente adorna la formación de las especialidades de Maestros Araño, C. (2008:189), en la universidad española el tipo de enseñanza que se ha llevando a cabo para le Educación Artística ha sido eminentemente práctico, frente la consideración conceptual de disciplinas afines como la Estética o la Historia del Arte, lo que genera formas de intervención educativa diferentes y que proyectos curriculares oficiales no permiten conciliar Martínez, L. Ma.; Gutiérrez, R.; Escaño, C. (coords.) (2008:12). La educación artística y su área de enseñanza ha sido fruto de un intenso debate entre los profesionales españoles que la desarrollan y los de otras áreas cercanas como el arte, de las ciencias humanas y sociales, Marín (2000c:153), se decanta por la denominación didáctica de la expresión plástica, en la que integra la educción artística y estética, es decir, los procesos de enseñanza y aprendizaje de las Artes, las Culturas y las Comunicaciones Visuales. Por otra parte, si atendemos a la denominación que hacen del área los principales idiomas de prestigio académico, es Art Education, Education Artistique o Arte-Educação, y aunque no es la única designación en Europa, pues convive con la de Didáctica de la Plástica, Educación Visual, Cultura Visual, etc., si es predominante en el mundo angloamericano, por lo que consideramos que Educación Artística, se ajusta más a la corriente internacional, aunque si atendemos al lenguaje legislativo sobre el nombre de las materias y su enseñanza, la mayoría utiliza la fórmula didáctica de la materia en cuestión, así, didáctica de la expresión plástica, las polémicas sobre la denominación del área, es un síntoma de los cambios y debates que se dan en la disciplina, algunos miembros del área de conocimiento que nos ocupa, no se sienten identificados con el nombre del área, Didáctica de la Expresión Plástica, por el hecho, según ellos, de haber quedado obsoleta en su significado o acepción, y por resultar excesivamente estricta en su descripción del ideario y en referencia al corpus en cual hoy en día se han instalado Huerta, R. (2001:12). Más allá de esta discusión inicial sobre la denominación, es importante constatar que tipo de enseñanza se esta llevando a cabo, la 
mayoría de los especialistas en el Área de Didáctica de la Expresión Plástica planteaban ante la implantación de los nuevos Grados en Magisterio, la necesidad de promover un itinerario formativo en "Educación Artística y Lenguajes Audiovisuales" dentro del título de Grado, como recoge Gutiérrez, R. (2008:31), y otros profesionales de la Educación Artística que se reunieron con ocasión de las Jornadas sobre Educación Artística en 2005 y que a modo de conclusión se recogieron en el Informe sobre la situación de la Educción de las Artes Visuales y la Formación Inicial de sus docentes VV.AA. (2005), en este informe se reivindicaba la implantación de dicho itinerario en artes, las universidades que han llegado a implantar este mención se encuentran ahora en un contradicción, el informe sobre la situación de la Educción de las Artes Visuales y el itinerario en "Educación Artística y Lenguajes Audiovisuales", estaba apoyado por profesionales de la Educación Artística y por la Conferencia de Decanos y Directores de Centros de Magisterio AA.VV. Op. cit. y Maldonado, A. (2002) en Gutiérrez, R. (2008:32).

Tomando como referencia el marco contextual del Espacio Europeo de Educación Superior (EEES), en los últimos años se ha hecho en la Universidad española una importante labor de adaptación a los presupuestos de Bolonia y que aún se sigue haciendo, para la completa implantación del nuevo modelo educativo con la implantación de los nuevo Grado de Maestro en Educación Infantil y Grado de Maestro en Educación Primaria. La institución de los nuevos títulos de Grado, supone una reorganización completa del diseño de los estudios. Esto supone que hay que hacer una minuciosa selección y distribución de las competencias, entre generales y específicas de cada carrera, que se van a trabajar y evaluar en cada asignatura, intentando que no se repitan en demasía a lo largo de los cursos. Por tanto, los objetivos o resultados esperados a conseguir en cada asignatura, materia o módulo tiene que estar relacionados directamente con las competencias seleccionadas para cada uno de ellos, teniendo en cuenta que una competencia puede relacionarse con varios resultados de aprendizaje o un resultado de aprendizaje con varias competencias, y que las actividades deben planificarse en función de las competencias y los resultados de aprendizaje, ajustando la carga de trabajo de los alumnos a los porcentajes establecidos en los diferentes módulos, materias y asignaturas. Para conseguir estos presupuestos, se realizan diferentes iniciativas. Por un lado se está haciendo una importante labor de coordinación entre los nuevos grados y las diplomaturas a extinguir. Esto significa que se tiene en cuenta la distribución de competencias y los niveles de adquisición de las mismas a lo largo de las asignaturas, módulos y materias de los que constan los estudios, teniendo especialmente cuidado en los del nuevo grado, los cuales quedan plasmados en los criterios de evaluación y en los diferentes tipos actividades propuestas para la adquisición de las competencias. A su vez, se marca la distribución del tiempo dedicado al trabajo presencial, guiado o autónomo y las formas de evaluar dicho trabajo, que, es una evaluación continua.

\section{Conclusión}

La relación entre los resultados de aprendizaje por proyectos con los contenidos y actividades propuestas por el docente, permite evaluar el conocimiento, evaluar la compresión, mediante recursos y materiales para la educación plástica, evaluar la experimentación con técnicas, procedimientos y recursos y por último, evaluar la adquisición de estrategias y métodos didácticos. El docente de expresión plástica y visual, basa su metodología en problemas a solucionar para que el alumno trabaje el proyecto en un tiempo determinado, en pequeños grupos, lo que fomenta la participación. El método por proyectos enfrenta al alumno a situaciones que lo llevan a resolver problemas, además, en ellos la espontaneidad será un recurso más del área, lo que recalca la importancia de aplicar metodologías activas con el alumno a través de la investigación-descubrimiento, por otra parte, es importante facilitar recursos al alumno para motivarlo y que los reproduzca en su futura docencia, además de fomentar la capacidad del alumno para planificar proyectos más allá del aula y potenciar un cambio en los procesos del aprendizaje significativo. El área de didáctica de la expresión plástica, no ha estado al margen de los cambios educativos en los grados de infantil y primaria, actualmente, la educación artística posee unas características especiales que la diferencian de otras disciplinas, por ello, su desarrollo mediante competencias es un instrumento al servicio del profesorado para la renovación de su práctica, el aprendizaje debe caracterizarse por ser funcional, es decir, que lo aprendido pueda ser utilizado realmente cuando las circunstancias de la vida lo exijan. Perales Molada, R. y Montijano Serrano, B. (2008:79). 
$2^{\text {nd }}$ International Conference Art, Illustration and Visual Culture in Infant and Primary Education $2^{\circ}$ Congresso Internacional

de Arte, Ilustração e Cultura Visual

na Educação Infantil e Primária

Las competencias específicas del docente de plástica, es un pilar importante en la formación del futuro maestro de infantil y primaria, con ellas se busca incentivar la calidad las enseñanzas de educación artística y diseñar un plan de ordenación de la docencia, estrechamente relacionado con la investigación educativa basada en las artes visuales. En una docencia de calidad en artes visuales deben proporcionar al estudiante las herramientas necesarias para insertarse en la sociedad y en la producción de investigación. La docencia en artes visuales abre el campo profesional de los futuros graduados, en la educación formal y en la no formal, como en museos y otras instituciones donde es valorada la acción educativa. Exigencia por parte del profesorado y del estudiante en potenciar el mayor rendimiento académico basado en la educación artística y su implementación en la docencia. Las competencias específicas de plástica son una pieza clave de los nuevos grados de Maestro en Educación Infantil y Primaria y sobre ella debe pivotar la docencia y la creación de conocimiento que obligatoriamente deberá repercutir en la formación del maestro. Es importante potenciar la investigación en educación artística desde los primeros niveles universitarios, para que constituya una parte más del quehacer habitual del maestro de plástica, para ello es necesario incorporar al alumno a grupos de investigación educativa. El proyecto de investigación en artes visuales como herramienta docente que permita a los alumnos enfrentarse al estudio de diferentes casos y su resolución plástica. Potenciar la presencia de la Educación Artística en trabajos interdisciplinares de la Facultad de Educación y de otras áreas. La transferencia de conocimientos sobre educación artística es una herramienta fundamental de crecimiento y de desarrollo de la sociedad y debe ser el área de didáctica de la expresión plástica la que genere este conocimiento y lo difunda. Programas de prácticas en museos y otras instituciones que dispongan de un departamento de educación y acción cultural, lo que permite la transferencia de conocimientos, en ambos sentidos desde la educación formal y no formal. Plan de apoyo a la transferencia de resultados de investigación en artes visuales. Apoyo institucional para incrementar, por su importancia, la presencia de la educación artística en los currículos de Maestro en Infantil y Primaria.

\section{Referencias bibliográficas}

Acaso, M. (2009): La educación artística no son manualidades. Nuevas prácticas en la enseñanza de las artes y la cultura visual, Catarata, Madrid.

Aguirre Arriaga, I. (2000a): Teorías y prácticas en Educación Artística, Universidad Pública de Navarra, Pamplona.

Araño Gisbert, J.C. (1994): “Arte, educación y creatividad”, en: Pixel-Bit: Revista de medios y educción, № 2.

Arañó, J.C., Mañero, A. (coords.). (2003): Actas del Congreso INARS: la investigación en las artes plásticas y visuales, Universidad de Sevilla, Secretariado de Publicaciones.

Barbosa, Ana Mãe (coord.) (2002): Inquietações e Mudanças no Ensino da Arte, Cortez Editora, São Paulo.

Escaño, C. (1999): "Arte en la era audiovisual: replicantes, realidades”, en: Arte, individuo y sociedad, Nº 11, $(61-68)$.

Gutiérrez Pérez, R. (2008): “Especialistas o generalistas?. La formación artística del profesorado de educación infantil y primaria” en: Martínez, L. Ma. (coord.): Nuevas propuestas de acción en educación educativa, Universidad de Málaga, Servicio de Publicaciones, (25-36).

Ley Orgánica de Educación (2006): Boletín Oficial del Estado, 3 de mayo

Ley Orgánica de Universidades (2001): Boletín Oficial del Estado, 24 de diciembre

Maeso, F. y González, Ma.R. (2005): "El valor de la motivación en la educación artística con personas mayores", en: Arte, individuo y sociedad, No 17, (43-60).

Marín, R. (2003) (Coord.): Didáctica de la Educación artística para primaria, Pearson Prentice Hall, Madrid.

Martínez, L. Ma.; Gutiérrez, R.; Escaño, C. (coords.) (2008): Nuevas propuestas de acción en educación educativa, Universidad de Málaga, Servicio de Publicaciones. 